\title{
STUDIES ON BIS(PARA ANISIDINE ACETYLACETONATO) NICKEL (II) COMPLEX
}

\author{
H. N. Aliyu and U. L. Bilyamin \\ Department of Chemistry Bayero University, P. M. B. 3011, Kano, Nigeria \\ Correspondence uthor: hnuhu2000@yahoo.com
}

\begin{abstract}
Schiff base was prepared from the reaction of para anisidine and acetylacetone. Bis(para anisidineacetylacetonato)nickel(II) complex was synthesized by the reaction of the prepared para anisidineacetylacetone ligand and nickel(II) chloride. The prepared Schiff base is orange yellow, has a yield of $61.90 \%$ and melting point of $86^{\circ} \mathrm{C}$. The complex prepared is red, has a yield of $71 \%$ and decomposition temperature of $270^{\circ} \mathrm{C}$. The para anisidineacetylacetone ligand is soluble in water and most organic solvents, similarly, its nickel(II) complex compound is soluble in most solvents except water and methanol, in which it slightly soluble. The molar conductance of bis(para anisidineacetylacetonato)nickel (II) complex compound determined is $4.70 \mathrm{ohm}^{-1} \mathrm{~cm}^{2} \mathrm{~mol}^{1}$, suggesting that the complex is non-electrolyte. The infra-red spectral data of para anisidine and acetylacetone base showed a medium size band in the range $1595-1630 \mathrm{~cm}^{-1}$, attributable to $v(C=N)$ stretching vibrations, the same band is observable in the nickel(II) complex, indicating coordination of the ligand to the nickel(II) ion. The broad band in the range $3257-3420 \mathrm{~cm}^{-1}$ observed in para anisidineacetylacetone ligand is assigned to $\mathrm{v}(\mathrm{O}-\mathrm{H})$ stretching vibrations, which is absent in anisidineacetylacetonato)nickel(II) complex, as a result of deprotonation of the para anisidineacetylacetone on coordination to the nickel(II) ion. The dissociation constant (pKa) of para-anisidine acetylacetone determined is 12.66. The stability constant of anisidineacetylacetonato)nickel (II) complex compound determined is $1.29 \times 10^{6}$ and the corresponding Gibb's free energy of complex is $-3.48 \times 10^{4} \mathrm{KJ}$ per mole. The average number of para anisidine ligand coordinated to nickel(II) ion determined using potentiometer is 2, suggesting a four coordinate complex compound.
\end{abstract}

Keywords: Anisidineacetylacetone, complex compound, stability constant, potentiometry.

\section{INTRODUCTION}

Anisidineacetylacetone ligand belongs to a class of chemical compounds called, Schiff bases, which are compounds containing the azomethine $(-\mathrm{C}=\mathrm{N}-)$ group. They are usually obtained by the condensation of an aldehyde or ketone with primary amine (Holm et al., 1966). Schiff base is a nitrogen analog of an aldehyde or ketone in which the $\mathrm{C}=\mathrm{O}$ group is replaced by a carbon - nitrogen double bond $(\mathrm{C}=\mathrm{N})$ with the nitrogen atom connected to an aryl or alkyl group (Schiff bases are of the general formula $\mathrm{R}_{1} \mathrm{R}_{2} \mathrm{C}=\mathrm{NR}_{3}$, where $R_{3}$ is an aryl or alkyl group that makes the Schiff base a stable imine (Henry and Lange, 1977). Schiff base transition metal complexes have been known, however, there was no comprehensive, systematic study until the preparative work of Pfeiffer and associates (1931), in which they reported a series of complexes derived from Schiff bases of salicylaldehyde and its substitution products. Delephine (1898) prepared complexes by reacting metal acetate, salicyladehyde and a primary amine in alcohol and demonstrated 2:1 stoichiometry (Holm et al., 1966). Recently, Gupta et al. (2002) reported the synthesis and characterization of cobalt (II) $\mathrm{N}, \mathrm{N}^{\prime}-$ bis(acetylacetone)ethylenediiminato complex from the interaction of cobalt(II) salt and $\mathrm{N}$, N'bis(acetylacetone)ethylenediimine Schiff base ligand. In another report, Xishi et al. (2003) described the synthesis and spectroscopic properties of manganese(II), cobalt(II) and copper(II) complexes with novel Schiff base ligand derived from 2, 2' bis(pmethoxylphenylamine) and salicylic aldehyde.

Schiff bases and their complexes have been studied for their important properties, for example, thiazole and benzothiazole Schiff bases show effective antifungal activity (Dash et al., 1984). Some Schiff bases derived from quinazolinones show antifungal activity against Candida albicans, Trichophyton rubrum, T. mentagrophytes, A. niger and Microsporum gypseum (Rao et al., 1987). Metal complexes of molybdenum(IV) and manganese(II) with Schiff base ligands derived from hydrazine carboxamide and hydrazine carbothiamide show antibacterial activity against $S$. aureus and xanthomonas compestris (Singn et al., 1999). In another report, Guar (2003) explained that Schiff base complexes of copper(II) and nickel(II) show antibacterial activities to Colibacillus and Pseudomonas aeruginosa. This paper reports the studies on bis(para anisidine acetylacetonato) nickel(II) Complex.

\section{MATERIALS AND METHODS}

In the preparation of reagents, chemical of analytical grade purity and distilled water were used. All glass wares were well washed with detergent rinsed with distilled water and dried in an oven before use. 
All weighings were carried out on electric metler balance model $A B 54$ of 0.001 sensitivity. IR spectral analyses were recorded using as Fourier transformed IR Genesis series model in Nujol in the range $4000-$ $400 \mathrm{~cm}^{-1}$. $\mathrm{pH}$ measurements were carried out using Jenway $\mathrm{pH}$ meter model 3320 and electrical conductivity using Jenway conductivity meter model 4010.

\section{Preparation of para-Anisidine Acetylacetone}

To a $500 \mathrm{~cm}^{3}$ round bottom flask were added $12.3 \mathrm{~g}$ recrystallized para- anisidine in $20 \mathrm{~cm}^{3}$ of ethanol, $10.2 \mathrm{~cm}^{3}$ of acetylacetone in $20 \mathrm{~cm}^{3}$ of ethanol and a few drops of piperidine as condensing agent. The mixture on ice cooling gave an orange - yellow semisolid which was preserved in a desiccator (Raman, et al, 2003).

\section{Preparation of Bis(para anisidine acetylacetonato) Nickel(II) Complex}

$2.05 \mathrm{~g}(0.01 \mathrm{M})$ of the ligand was dissolved in $20 \mathrm{~cm}^{3}$ of ethanol. This was mixed with 0.05 mole of the nickel(II) chloride in $20 \mathrm{~cm}^{3}$ of ethanol. The mixture was refluxed for two (2) hours on a water bath and then concentrated and then cooled to $0^{\circ} \mathrm{C}$. The Solid product obtained was filtered and washed with ethanol and dried (Raman et al., 2003).

Determination of Dissociation Constant of para-Anisidine Acetylacetone

To a $400 \mathrm{~cm}^{3}$ beaker were added $90 \mathrm{~cm}^{3}$ of distilled water, $100 \mathrm{~cm}^{3} 0.2 \mathrm{M} \mathrm{KNO}_{3}, 10 \mathrm{~cm}^{3}$ of $0.4 \mathrm{M}$ paraanisidine acetylacetone and a magnetic stirring bar before the electrodes of a standardized meter were introduced. A $10 \mathrm{~cm}^{3}$ of standardized aqueous $\mathrm{NaOH}$ was added dropwise with stirring. After each $0.5 \mathrm{~cm}^{3}$ addition, the amount of $\mathrm{NaOH}$ solution and the corresponding $\mathrm{pH}$ were recorded. Points in the 20 to 80 percent titration range were used to calculate the pKa (Gregory et al., 1978).

Determination of Stability Constant of Bis(paraanisidine acetylacetonato) Nickel(II) complex Into a $400 \mathrm{~cm}^{3}$ beaker were added $90 \mathrm{~cm}^{3}$ of distilled water, $100 \mathrm{~cm}^{3} 0.2 \mathrm{M} \mathrm{KNO}_{3}, 10 \mathrm{~cm}^{3}$ of $0.1 \mathrm{M} \mathrm{HNO}_{3}$ acid, and one millimole of nickel(II) chloride tetrahydrate, respectively. $0.2 \mathrm{~cm}^{3}$ of $0.4 \mathrm{M}$ sodium salt of para anisidine acetylacetonate was added and after each addition with stirring the corresponding $\mathrm{pH}$ reading was recorded. The addition of the sodium salt of para anisidine acetylacetonate solution was continued until full $10 \mathrm{~cm}^{3}$ was added. The sodium salt of para anisidine acetylacetone was prepared by exactly neutralizing a weighed solid para anisidine acetylacetone with a calculated amount of standardized $0.48 \mathrm{M} \mathrm{NaOH}$ and diluting the solution with dimethylsulphoxide to a total volume of $20 \mathrm{~cm}^{3}$ (Angelici, 1977).

\section{RESULTS AND DISCUSSION}

The reaction of acetylacetone and para anisidine formed an orange yellow crystalline para anisidine acetylacetone Schiff base of melting point of $86^{\circ} \mathrm{C}$ and $61.9 \%$ yield (Table 1 ). The reaction of para anisidine acetylacetone Schiff base and nickel (II) chloride yielded a crystalline red bis(para anisidine acetylacetonato) nickel(II) complex. The decomposition temperature of the complex compound is $270^{\circ} \mathrm{C}$, which is quite high, suggesting a stable compound (Table 1). The para anisidineacetylacetone Schiff base is soluble in water and most organic solvents, similarly, its nickel(II) complex compound is soluble in most solvents except water and methanol, in which it is slightly soluble. The molar conductance of the complex compound determined is $4.70 \mathrm{ohm}^{-1}$ $\mathrm{cm}^{2} \mathrm{~mol}^{-1}$, revealing that the complex compound is non-electrolyte (Table 2). The infra-red spectral analysis of para anisidine schiff base showed a band in the range $1595-1630 \mathrm{~cm}^{-1}$, attributable to $V(C=N)$ stretching vibrations, the same band is observable in the complex formed by the Schiff base with cobalt(II) ion, indicating its coordination to the metal ion ((Jezowska et al, 1988; Abdulsamath et al., 1992). The far infrared spectra of the complex showed strong bands at $535 \mathrm{~cm}^{-1}$ and $352 \mathrm{~cm}^{-1}$, which are assigned to $\checkmark(\mathrm{M}-\mathrm{O})$ and $V(\mathrm{M}-\mathrm{N})$ stretching vibrations respectively, confirming the coordination of the ligand to the nickel ion (Silverstein and Bassler, 1967; Koji, 1977). The broad band in the range $3257-3420 \mathrm{~cm}^{-1}$ observed in para-anisidine schiff base is assigned to $(\mathrm{O}-\mathrm{H})$ stretching vibrations, which is absent in the nickel(II) complex compound due to deprotonation of the Schiff base on its coordination to the nickel(II) ion (Table 4). The dissociation constant ( $\mathrm{pKa}$ ) of the Schiff base determined is 12.66 (Table 5). The ratio of nickel (II) ion to Schiff base determined potentiometrically is $1: 2$, implying that it is a four coordinate complex (Table 6). The stability constant of bis(para-anisidine acetylacetonato) nickel(II) complex compound determined is $1.29 \times 10^{6}$, and the corresponding Gibb's free energy of the nickel(II) Schiff base complex determined is $-3.48 \times 10^{4} \mathrm{~kJ} \mathrm{~mol}^{-1}$, which is very low indicating that the complex is quite stable (Table 7).

\begin{tabular}{|c|c|c|c|c|}
\hline Compound & Colour & $\begin{array}{l}\text { Melting Point } \\
\left({ }^{\circ} \mathrm{C}\right)\end{array}$ & $\begin{array}{l}\text { Decomposition } \\
\text { Temp. }\left({ }^{\circ} \mathrm{C}\right)\end{array}$ & $\begin{array}{c}\text { Percent Yield } \\
(\%)\end{array}$ \\
\hline Schiff base & Orange yellow & 86.0 & - & 61.9 \\
\hline$\left[\mathrm{NiL}_{2}\right]$ & Red & - & 270.0 & 71.0 \\
\hline
\end{tabular}

Key: $L=$ Anisidine acetylacetonato

Table 2: Solubility of the Schiff base and its nickel(II) complex in some solvents

\begin{tabular}{lccccccc}
\hline Compound & Water & Methanol & Ethanol & Acetone & Nitrobenzene & Chloroform & DMSO \\
\hline Schiff base & S & S & S & S & S & S & S \\
{$\left[\mathrm{NiL}_{2}\right]$} & SS & SS & S & S & S & S & S \\
\hline
\end{tabular}

Where $\mathrm{S}=$ soluble and $\mathrm{SS}=$ slightly soluble. 
Bajopas Volume 4 Number 1 June, 2011

From the analytical results of the complex compound, the structure below is proposed.<smiles></smiles>

Fig.1: Molecular formula of bis(para-anisidine acetylacetonato) nickel(II) complex

Table 3: Conductivity measurement of the Schiff base and its nickel(II) complex

\begin{tabular}{lc}
\hline Compound & Molar Conductance $\left(\mathrm{Ohm}^{-1} \mathbf{c m}^{\mathbf{2}} \mathbf{m o l}^{-\mathbf{1}}\right)$ \\
\hline$\left[\mathrm{NiL}_{2}\right]$ & 4.7
\end{tabular}

Table 4: Infra red spectral data of the Schiff base and its nickel(II) complex

\begin{tabular}{|c|c|c|c|c|}
\hline Compound & $V(C=N)\left(\mathrm{cm}^{-1}\right)$ & $v(0-\mathrm{H})\left(\mathrm{cm}^{-1}\right)$ & $V(M-0)\left(\mathrm{cm}^{-1}\right)$ & $V(M-N)\left(\mathrm{cm}^{-1}\right)$ \\
\hline Schiff base & $1595-1630$ & $3257-3420$ & - & - \\
\hline$\left[\mathrm{NiL}_{2}\right]$ & $1570-1600$ & - & 535 & 352 \\
\hline
\end{tabular}

Table 5: Determination of pKa of Para-anisidine acetylacetone

\begin{tabular}{|c|c|c|c|c|c|c|c|}
\hline S/No. & $\begin{array}{c}\text { Value of } \\
\mathrm{NaOH}\left(\mathrm{cm}^{3}\right)\end{array}$ & pH & $\begin{array}{c}10^{-12}\left[\mathrm{H}^{+}\right] \\
\text {moldm }^{-3}\end{array}$ & $\begin{array}{c}10^{-3}\left[\mathrm{OH}^{-}\right] \\
\text {moldm }^{-3}\end{array}$ & $10^{-3}\left[\mathrm{Na}^{+}\right]$, moldm $^{-3}$ & {$[A-]$, moldm $^{-3}$} & pKa \\
\hline 1 & 0.5 & 8.99 & 684.7000 & 2.005 & 1.47 & 0.0200 & 10.37 \\
\hline 2 & 1.0 & 10.46 & 23.2000 & 2.006 & 2.29 & 0.0199 & 11.52 \\
\hline 3 & 1.5 & 10.92 & 8.0445 & 2.008 & 3.42 & 0.0197 & 11.78 \\
\hline 4 & 2.0 & 11.13 & 4.9602 & 3.256 & 4.55 & 0.0198 & 11.89 \\
\hline 5 & 2.5 & 11.27 & 3.5933 & 4.495 & 5.68 & 0.0197 & 12.03 \\
\hline 6 & 3.0 & 11.38 & 2.7890 & 5.790 & 6.80 & 0.0197 & 12.18 \\
\hline 7 & 3.5 & 11.46 & 2.3200 & 6.962 & 7.90 & 0.0197 & 12.34 \\
\hline 8 & 4.0 & 11.54 & 1.9297 & 8.370 & 9.00 & 0.0196 & 12.38 \\
\hline 9 & 4.5 & 11.60 & 1.6807 & 9.610 & 1.00 & 0.0196 & 12.39 \\
\hline 10 & 5.0 & 11.65 & 1.4980 & 10.782 & 11.22 & 0.0195 & 12.47 \\
\hline 11 & 5.5 & 11.70 & 1.3351 & 12.098 & 12.51 & 0.0195 & 12.55 \\
\hline 12 & 6.0 & 11.74 & 1.2176 & 13.265 & 13.40 & 0.0194 & 12.68 \\
\hline 13 & 6.5 & 11.77 & 1.1363 & 14.213 & 14.48 & 0.0194 & 12.85 \\
\hline 14 & 7.0 & 11.80 & 1.0605 & 15.230 & 15.56 & 0.0193 & 12.92 \\
\hline 15 & 7.5 & 11.83 & 0.9897 & 16.319 & 16.63 & 0.0193 & 13.11 \\
\hline 16 & 8.0 & 11.86 & 0.8821 & 17.486 & 17.70 & 0.0192 & 13.29 \\
\hline 17 & 8.5 & 11.88 & 0.8821 & 18.311 & 18.75 & 0.0192 & 13.69 \\
\hline 18 & 9.0 & 11.91 & 0.2320 & 19.620 & 20.31 & 0.0191 & 13.98 \\
\hline 19 & 9.5 & 11.94 & 0.7683 & 21.020 & 21.69 & 0.0191 & 14.17 \\
\hline 20 & 10.0 & 11.96 & 0.7337 & 22.010 & 22.60 & 0.0190 & 14.54 \\
\hline
\end{tabular}

Average dissociation constant of Para-anisidine acetylacetone Pka $=12.66$ 
Table 6: Determination of the number of Schiff base (para-anisidine acetylacetone) chelated (n) to nickel(II) ion

\begin{tabular}{|c|c|c|c|c|c|c|c|c|}
\hline S/No. & $\begin{array}{l}\text { Vol. of sodium } \\
\text { salt Schiff base }\left(\mathrm{cm}^{3}\right)\end{array}$ & pH & $\begin{array}{l}10^{-3}\left[\mathrm{H}^{+}\right] \\
\text {moldm }\end{array}$ & $\begin{array}{l}10^{-12}\left[\mathrm{OH}^{-}\right] \\
\text {moldm }^{-3}\end{array}$ & $\begin{array}{l}10^{-3} A_{\text {tot, }} \\
\text { moldm }^{-3}\end{array}$ & $\log \left[A^{-}\right]$ & $\begin{array}{l}10^{-3} \mathrm{M}_{\text {tot, }} \\
\text { moldm }^{-3}\end{array}$ & $\mathbf{n}$ \\
\hline 1 & 4.2 & 3.68 & 1.40 & 11.55 & 8.23 & -3.08 & 4.90 & 0.6 \\
\hline 2 & 4.4 & 3.70 & 1.34 & 12.10 & 8.61 & -3.06 & 4.89 & 0.8 \\
\hline 3 & 4.6 & 3.72 & 1.28 & 12.67 & 8.99 & -3.03 & 4.89 & 0.8 \\
\hline 4 & 4.8 & 3.74 & 1.22 & 13.27 & 9.38 & -3.01 & 4.88 & 0.9 \\
\hline 5 & 5.0 & 3.76 & 1.16 & 13.89 & 9.76 & -2.99 & 4.88 & 1.0 \\
\hline 6 & 5.2 & 3.79 & 1.09 & 14.88 & 10.14 & -2.98 & 4.87 & 1.1 \\
\hline 7 & 5.4 & 3.81 & 1.04 & 15.59 & 10.52 & -2.96 & 4.87 & 1.2 \\
\hline 8 & 5.6 & 3.83 & 0.99 & 16.32 & 10.89 & -2.94 & 4.86 & 1.2 \\
\hline 9 & 5.8 & 3.85 & 0.95 & 17.09 & 11.27 & -2.93 & 4.86 & 1.3 \\
\hline 10 & 6.0 & 3.87 & 0.90 & 17.89 & 11.65 & -2.92 & 4.85 & 1.4 \\
\hline 11 & 6.2 & 3.90 & 0.84 & 19.17 & 12.03 & -2.91 & 4.85 & 1.5 \\
\hline 12 & 6.4 & 3.92 & 0.80 & 20.08 & 12.40 & -2.89 & 4.85 & 1.6 \\
\hline 13 & 6.6 & 3.94 & 0.77 & 21.02 & 12.78 & -2.87 & 4.84 & 1.6 \\
\hline 14 & 6.8 & 3.96 & 0.75 & 22.01 & 13.15 & -2.86 & 4.84 & 1.7 \\
\hline 15 & 7.0 & 3.98 & 0.70 & 23.05 & 13.53 & -2.85 & 4.83 & 1.8 \\
\hline 16 & 7.2 & 4.00 & 0.67 & 24.14 & 13.90 & -2.83 & 4.83 & 1.9 \\
\hline 17 & 7.4 & 4.02 & 0.64 & 25.28 & 14.27 & -2.82 & 4.82 & 2.0 \\
\hline 18 & 7.6 & 4.04 & 0.61 & 26.47 & 14.64 & -2.81 & 4.82 & 2.0 \\
\hline 19 & 7.8 & 4.06 & 0.58 & 27.71 & 15.01 & -2.80 & 4.81 & 2.1 \\
\hline 20 & 8.0 & 4.08 & 0.56 & 29.02 & 15.39 & -2.79 & 4.81 & 2.2 \\
\hline 21 & 8.2 & 4.11 & 0.52 & 30.91 & 16.12 & -2.78 & 4.80 & 2.4 \\
\hline 22 & 8.4 & 4.13 & 0.50 & 32.38 & 16.53 & -2.77 & 4.79 & 2.5 \\
\hline 23 & 8.6 & 4.15 & 0.50 & 34.86 & 16.87 & -2.76 & 4.79 & 2.5 \\
\hline 24 & 8.8 & 4.17 & 0.47 & 36.93 & 17.23 & -2.74 & 4.78 & 2.6 \\
\hline 25 & 9.0 & 4.19 & 0.45 & 38.21 & 17.60 & -2.73 & 4.78 & 2.7 \\
\hline 26 & 9.2 & 4.21 & 0.43 & 40.32 & 18.02 & -2.72 & 4.78 & 2.8 \\
\hline 27 & 9.4 & 4.23 & 0.41 & 42.63 & 18.32 & -2.72 & 4.77 & 2.8 \\
\hline 28 & 9.6 & 4.25 & 0.40 & 44.73 & 18.68 & -2.71 & 4.76 & 2.9 \\
\hline 29 & 9.8 & 4.26 & 0.36 & 46.21 & 19.07 & -2.69 & 4.76 & 3.0 \\
\hline 30 & 10.0 & 4.26 & 0.36 & 48.66 & 19.21 & -2.78 & 4.75 & 3.0 \\
\hline
\end{tabular}

Where $A_{\text {tot }}=$ Total concentration of the dissociated ligand, $M_{\text {tot }}=$ Total concentration of the metal salt added. $\mathrm{n}=$ Average number of coordinated Schiff base (2.0)

Table 7: Stepwise formation and the overall stability constants of the complex

\begin{tabular}{|c|c|c|c|c|}
\hline Compound & Stepwise & $y$ constant & Overall stability constant & Gibb's free energy \\
\hline & $K_{1}$ & $K_{2}$ & $\mathbf{K}_{\mathrm{f}}$ & $\Delta G\left(k J \mathrm{Mol}^{+}\right)$ \\
\hline$\left[\mathrm{NiL}_{2}\right]$ & $1.589 \times 10^{3}$ & $8.13 \times 10^{2}$ & $1.291 \times 10^{6}$ & $-3.48 \times 10^{4}$ \\
\hline
\end{tabular}

\section{REFERENCES}

Abdulsamath S., Raman M., Raman N., (1992), Complexes of Copper (II) cinnamalidene Transition Metal Chem. 17:13

Angelici, R. J. (1977). Synthesis and techniques in inorganic chemistry W. B. Saunders company, $2^{\text {nd }}$ Edition Philadelphia, Pp $115-127$.

Dash B., Mahapatra P. K., and Patnaik J. M., (1984). Fungicidal activities of Schiff base derived from $p$ hydroxobenzaldehydes and their derivatives, J. Ind. Chem. Soc., 61(2): $1061-1064$.

Gregory, S., Thomas, B. R., Robert, J. A. (1978) "Synthesis and Techniques in Inorganic Chemistry" $3^{\text {rd }}$ edition, Longman Publishers, London, 119-126.

Guar S. (2003). Physico-chemical and biological properties of manganese (II), cobalt (II), nickel (II) and copper(II) chelates of Schiff bases, Asian Journal of chemistry, 15(2): 250-254.

Gupta S.K. Hitchcock and Kushwah Y.S. (2002), Synthesis, characterization and crystal structure of a $\mathrm{Ni}(\mathrm{II})$ Schiff base complex derived from acetylacetone and ethylene diamine; J. of Coord. Chem...,55 (12), 1401-1407.

Henry P. M. and Lange G. L. (1977), The chemistry of double bonded functional groups, S. Patai , Interscience, New York, suppl. A, part 2, Pp 1067.

Holm R. H., Everett G. W., and Chakravorty A. (1966), Metal complexes of Schiff bases and $\beta$ - ketoimines, Progr. Inorg. Chem., 7: $83-214$.
Jezowska, B. Lisowski, J. and Chemielewski P. (1988), Synthesis and spectroscopic studies of Schiff base complexes polyhedron, 7(5), 337-340.

Koji N., (1977), Infrared Absorption Spectroscopy, $2^{\text {nd }}$ Edition, Holden-Day Inc, Sanfrancisco, 20-49

Pfeiffer P., Buchholz E. and Bauer O., (1931). Inner complex salts from hydroxyaldimines and hydroxyketimines, J Prakt. Chm., 129, $163-177$.

Raman, N. Veeraraj, A. Sami P. (2003) "Copper (II) Complex of 3-cinnamalideneacetylacetone" Proc. Indian Academic of Sci. 112, No. 5, 515-521.

Rao N. R., Rao P. V., Reddy G. V. and Ganorkar M. C., (1987), Metal chelates of a physiologically active O:N:S tridentate Schiff base, Indian J. Chem., 26(1): 887 -890.

Silverstein, S. M. and Bassler, G. C., (1967); "Spectrophotometric Identification of Organic Compounds", Willey, New York, pp. 12-23.

Singh R., Gupta N. and Fahmi S, (1999). Biochemical aspects of dioxomolybdenum (IV) and manganese (II) complexes, Ind. Journ. of Chem, 38A: 1150-1158.

Xishi, T., Xian, H. Y., Qiang, C. and Minyu, T., (2003), "Synthesis of Some Transition Metal Complexes of a Novel Schiff Base Ligand Derived from 2, 2' - Bis(pMethoxy Phenylamine) and Salicylic Aldehyde", Molecules, 8: 439-443. 